results demonstrated that male gender, age less than one year at diagnosis, lower grade of reflux at presentation and unilateral reflux were all predictors of spontaneous resolution, which is consistent with previous studies. In addition, they were able to demonstrate that reflux improvement on imaging one year after diagnosis, as well as improvement in reflux grade from the previous year at any point during follow-up, were both independent predictors of resolution.

This study has important clinical implications when counseling with parents over the decision to continue waiting for spontaneous resolution versus pursuing surgical correction of reflux. Though many of us have assumed that improvement in the grade of reflux is a positive indicator of a greater likelihood for spontaneous resolution, we now have data to back up our assumptions.

Dr. M. Chad Wallis

Division of Pediatric Urology

University of Utah

Salt Lake City, Utah, USA

E-mail: chad.wallis@hsc.utah.edu

doi: 10.1590/S1677-553820100002000031

\title{
Incidence of new onset metabolic acidosis following enteroplasty for myelomeningocele
}

Adams RC, Vachha B, Samuelson ML, Keefover-Hicks A, Snodgrass WT

Department of Pediatrics, University of Texas Southwestern Medical Center at Dallas, Dallas, Texas, USA

J Urol. 2010; 183: 302-5

Purpose: Extant literature is mixed regarding risk of metabolic acidosis after enteroplasty for myelomeningocele. This study is the first known attempt to describe the pattern of developing metabolic acidosis in a group of children who underwent enteroplasty and served as their own controls. Multiple preoperative and postoperative laboratory measures for each child were obtained for comparison.

Materials and Methods: This retrospective cohort study allowed participants to serve as their own controls for pre-intervention and post-intervention analysis. The setting was a tertiary, university affiliated, interdisciplinary spina bifida program. All patients followed in the spina bifida program who had undergone ileal or colonic enteroplasty were included for review (total 113). Strict exclusion criteria were preoperatively diagnosed renal insufficiency, preexisting metabolic acidosis consistent with renal tubular acidosis ( $\mathrm{pH}$ less than 7.35, bicarbonate $20 \mathrm{mmol} / \mathrm{l}$ or less) and history of augmentation using gastric or ureteral tissue. Final analysis included 71 children who met inclusion criteria. Children in our spina bifida program periodically undergo routine laboratory evaluation of electrolytes, blood urea nitrogen, creatinine, blood count, and venous blood gases including $\mathrm{pH}$, bicarbonate and partial pressure of carbon dioxide. Primary outcome measures were comparative shifts in blood gases and electrolytes that would confirm the new onset of metabolic acidosis after enteroplasty. Changes in electrolytes and serum creatinine were secondary outcome measures to identify potential markers for postoperative effects. With each child as his/her own control, analysis included paired t tests.

Results: No statistically significant differences $(\mathrm{p}<0.05)$ were found when comparing laboratory values before and after bladder augmentation, including $\mathrm{pH}$, bicarbonate, partial pressure of carbon dioxide and electrolytes. No child had metabolic acidosis based on the aforementioned criteria. Followup ranged from 1 to 138 months after enteroplasty (mean 46.8). Respiratory compensation was considered in the analysis, and no difference in partial pressure of carbon dioxide following surgery was noted $(\mathrm{p}=0.65)$.

Conclusions: To our knowledge no previous study has examined the matched paired results of before and after development of metabolic acidosis among children (serving as their own controls) with myelomeningocele 
undergoing ileal or colonic enteroplasty. The negative statistical results in this controlled cohort are clinically significant. If a child with myelomeningocele has metabolic acidosis after enteroplasty, other clinical reasons beyond the effects of surgery warrant careful consideration.

\section{Editorial Comment}

The development of metabolic acidosis following enterocystoplasty is a common concern particularly in the pediatric population where such a procedure is intended to last a lifetime. The authors reviewed data from their spina bifida program that had undergone bladder augmentation using either ileum or colon. They excluded patients with preoperative renal insufficiency or metabolic acidosis. Patients in their spina bifida program routinely undergo serum evaluation of electrolytes, BUN, creatinine blood count, and venous blood gases giving them a unique opportunity to look at changes in serum values both before and after enterocystoplasty. They identified 71 children who met the inclusion criteria and had both preoperative and postoperative laboratory values. Their primary outcome was a shift in blood gases and electrolytes consistent with new onset of metabolic acidosis following bladder augmentations. Secondary outcomes included changes in electrolytes and serum creatinine. They found no evidence of new onset of metabolic acidosis following surgery for a mean follow-up period of almost four years.

Although concern for metabolic changes following enterocystoplasty in the pediatric population must be considered over extremely long time periods, it is interesting to note that these authors found no significant changes even after following their patients for a mean of four years. They also wisely point out that the spina bifida patient population is at risk for metabolic acidosis for other reasons including nutritional issues, chronic infection, pulmonary insufficiency, and/or renal insufficiency. As the authors point out in their conclusion, it may be just as important to consider other sources of metabolic acidosis (some of which may be correctable) rather than assuming the enterocystoplasty is to blame.

Dr. M. Chad Wallis

Division of Pediatric Urology University of Utah Salt Lake City, Utah, USA E-mail: chad.wallis@hsc.utah.edu 